\title{
Icariin Delays Brain Aging in Senescence-Accelerated Mouse Prone 8 (SAMP8) Model via Inhibiting Autophagy
}

\author{
Fa-Ju Chen, ${ }^{1}$ Bo Liu, ${ }^{1}$ Qin Wu, Jie Liu, Yun-Yan Xu, Shao-Yu Zhou, and Jing-Shan Shi \\ Key Laboratory of Basic Pharmacology of Ministry of Education and Joint International Research Laboratory of Ethnomedicine of \\ Ministry of Education, Zunyi Medical University, Zunyi, People's Republic of China
}

Received August 30, 2018; accepted February 6, 2019

\section{ABSTRACT}

Icariin (ICA), a major flavonoid extracted from the Chinese tonic herb Epimedium, exerts beneficial effects in a variety of age-dependent diseases, such as Alzheimer's disease (AD). However, the antiaging mechanisms remain unclear. The senescence-accelerated mouse-prone 8 (SAMP8) model has been used to study age-related neurodegenerative changes associated with aging and the pathogenesis of AD. Hence, the current study was designed to examine the effect of ICA on age-related cognitive decline in SAMP8 mice and explore the role of autophagy in the ICA-mediated neuroprotection. SAMP8 mice were administered with ICA starting at 5 months of age, and the treatment lasted for 3 consecutive months. Morris water maze was used to evaluate cognitive function. The senescenceassociated $\beta$-galactosidase staining was used to determine the number of senescence cells. The neuronal morphologic changes were examined via Nissl staining. The hippocampal neuronal ultrastructure was examined by transmission electron microscopy. The expression of autophagy protein was examined by Western blot. ICA-treated SAMP8 mice exhibited a robust improvement in spatial learning and memory function. Meanwhile, ICA reduced the number of senescence cells in the brains of SAMP8 mice, inhibited neuronal loss, and reversed neuronal structural changes in the hippocampi of SAMP8 mice. Moreover, ICA treatment also decreased the formation of autophagosomes in the hippocampus of SAMP8 mice, and reduced the expression of autophagy-related proteins LC3-II and p62. These results demonstrate that ICA possesses the ability to delay brain aging in SAMP8 mice, and the mechanisms are possibly mediated through the regulation of autophagy.

\section{Introduction}

Aging, a time-related deterioration of physiologic functions, occurs in all of the cells, tissues, and organs of an organism, leading to adverse changes, such as skin pigmentation, skin wrinkling, and organ senescence (López-Otín et al., 2013). Aging itself cannot be considered as a disease, but aging is the greatest risk factor for the functional decline of most organs (Barzilai et al., 2018). In particular, aging is accompanied by a variety of senile diseases, such as dementia (Deak et al., 2016). With the development of social economy and the improvement of medical and sanitary conditions, the elderly population is steadily on the increase. Demographic data show that $26.9 \%$ of the total population of China will be over 65 years old by 2050 , and China will be one of the countries in the world with the highest percentage of aged people (Fang et al., 2015). This will inevitably give rise to a host of socioeconomic challenges. Accordingly, there is a scientific urgency to study the mechanisms of aging and to develop effective antiaging drugs to

This work was supported by the National Natural Science Foundation of China [Grants 81773739 and 81160400]; Science and Technology Innovation Talent Team of Guizhou Province [Grant CJ-926]; Shijingshan's Tutor Studio of Pharmacology [GZS 2016(07)]; and a Construction of First-class Discipline project [GZS-2017(7)].

${ }^{1}$ F.-J.C. and B.L. contributed equally to this work.

https://doi.org/10.1124/jpet.118.253310. prevent age-related diseases. This is critical for nations such as China, which is experiencing rapid growth in the aging population.

In the course of aging, the brain is probably the most vulnerable organ in the process because of its high demand for oxygen, its limited ability to regenerate, and its low endogenous antioxidant capacity (Mecocci et al., 2018). Brain aging is accompanied by many pathologic changes and behavior abnormalities, such as the decline in cognitive function, brain atrophy, lipofuscinosis, $\beta$ amyloid deposition, and the loss of neurons (Serrano-Pozo et al., 2011). However, the mechanism of aging still remains elusive. Studies have suggested that autophagy is closely related to aging (Simonsen et al., 2008). Autophagy is a self-degradative process in which cytoplasmic contents such as long-lived proteins, pathogens, and damaged organelles are degraded by lysosomes (De Rechter et al., 2016). Autophagy thus plays a critical role in the prevention of neurodegenerative disorders, and dysfunction of autophagy affects cellular senescence and many other cellular signaling (Kang and Elledge, 2016). In addition, it has been demonstrated that autophagy plays an important role in the process of aging in the senescence-accelerated mouse prone 8 (SAMP8) model (Wang et al., 2017).

SAMP8, an animal model of aging, is an ideal model to study brain aging and dementia, and has been proposed as a mammal model to study rapid aging (Li et al., 2018). SAMP8

ABBREVIATIONS: ICA, icariin; LC3, microtubule-associated protein light-chain-3; MWM, Morris water maze; NS, normal saline; SA- $\beta$-gal, senescence-associated $\beta$-galactosidase; SAMP8, senescence-accelerated mouse prone 8; SAMR1, senescence-accelerated mouse resistant 1. 
mice present not only with Alzheimer's disease features, such as amyloid $\beta$ deposition and excessive phosphorylation of $\tau$ protein, but also have characteristics similar to those of aged humans, such as hair loss, shorter life span, reduced physical activity, and lordosis (Manich et al., 2011; Akiguchi et al., 2017). Thus, SAMP8 and the senescence-accelerated mouse resistant 1 (SAMR1) mice of the same genetic background are used to study senility. In recent years, several feasible strategies such as melatonin and caloric restriction have been studied to delay aging in SAMP8 mice, but they all have drawbacks (Cuesta et al., 2013; Garcia-Matas et al., 2015). Moreover, extracts from rosemary and sage, as well as antioxidants such as fish oil and $\alpha$ lipoic acid have also been shown to delay aging in SAMP8 mice (Tsuduki et al., 2011; Farr et al., 2012, 2016). With rich herbal medicine resources in China, we are searching for alternative approaches. In the present study, we employed SAMP8 and age-matched SAMR1 mice to study the beneficial effects of icariin [ $\mathrm{ICA}\left(\mathrm{C}_{33} \mathrm{H}_{40} \mathrm{O}_{15}\right)$ ].

ICA, a major flavonoid extracted from the Chinese tonic herb Epimedium, exerts beneficial effects in a multitude of age-dependent disease states, including bone loss, cancer, cardiovascular disease, and neurodegenerative disorders $(\mathrm{Li}$ et al., 2015a). We and other groups have found that ICA has anti-inflammatory, antioxidant, antiangiogenesis, and immunomodulation effects (Chen et al., 2014a; Li et al., 2015b; Wang et al., 2017). Additionally, our previous study (Guo et al., 2010) indicated that ICA improved spatial learning and memory abilities in lipopolysaccharide-induced cognitive dysfunction through the inhibition of hippocampus interleukin- $1 \beta$ and cyclooxygenase- 2 expression. It has also been reported that ICA improves cognitive impairments in SAMP8 mice via increasing monoamine levels, inhibiting oxidative damage and decreasing acetylcholinesterase activity (He et al., 2010). In addition, ICA can protect PC12 cells from oxygen-glucose deprivation and reperfusion-induced autophagy (Mo et al., 2016). However, whether the role of ICA in delaying aging in SAMP8 is related to the regulation of autophagy is unknown. Therefore, in the present study, we aimed to assess the preventative effects of ICA on SAMP8 mice, focusing on autophagy.

\section{Materials and Methods}

Materials and Animals. ICA (purity, >98\%) was supplied by Nanjing Zelang Medical Technology Co., Ltd (Nanjing, People's Republic of China). All reagents were reagent grade and commercially available.

Male SAMP8 and SAMR1 mice with infection and virus free were obtained from the Medical Department of Beijing University (Certificate number SCXK2016-0010). All mice were maintained in specific pathogen-free facilities of Zunyi Medical University (Certificate number SYXK 2014-003). Our specific pathogen-free facilities were tested once a year by the Chongqing Laboratory Animal Quality Testing Center (Chonqing, People's Republic of China). Mice were housed individually in the plastic cage with free access to food and water in a temperature- and humidity-controlled environment under a 12-hour light/dark cycle. All animal experiments were strictly carried out in accordance with National Institutes of Health Guidelines for the Care and Use of Laboratory Animals (National Institutes of Health Publication number 80-23, revised 1996), and the study protocol was approved by the Animal Use and Care Committee of Zunyi Medical University.

Experimental Design and Treatment. SAMP8 and agematched SAMR1 mice were randomly divided into the following five groups: SAMR1 group, SAMP8 group, and SAMP8 group plus ICA groups (SAMP8 receiving 20, 40, or $80 \mathrm{mg} / \mathrm{kg}$ ICA). Mice were administered normal saline (NS) or three doses of ICA orally once daily, starting at 5 months of age, and treatment lasted for 3 consecutive months. The doses and the duration of the treatment were based on our prior publications (Jin et al., 2014; Li et al., 2015). Four mouse right brains were fixed for histology, left hippocampal tissues were dissected for transmission electron microscopy, and the remaining brains were flash frozen for biologic analysis, including Western blot.

Morris Water Maze Test. The Morris water maze (MWM) test was carried out to evaluate the impact of ICA on the spatial learning and memory ability of mice, as described previously (Liu et al., 2018). The pool was divided into four quadrants, with a hidden platform located $1 \mathrm{~cm}$ below the water level in the center of the target quadrant. Before the experiment, all mice were allowed to swim freely for 120 seconds to adapt to the MWM. In this task, mice received four training sessions (one session/day) and a probe trial on the 5th day. Each session consisted of three trials with 2-hour intervals. A trial began when the mice was placed in the water at one of the three starting positions (excluding the platform quadrant), facing the wall. The swimming time of each mouse from the start location to reach the submerged platform (escape latency) was recorded. If the animal did not succeed, it was gently guided to the platform and left on it for 10 seconds, and the escape latency was recorded as 90 seconds. On the 5 th day, the platform was removed and the spatial probe test was carried out. The time spent in the target was measured by a computerbased video tracking system (Taimeng Co., Chengdu, People's Republic of China).

Senescence-Associated $\boldsymbol{\beta}$-Galactosidase Staining. Animals were sacrificed after the behavior tests, four mice from each group were perfused transcardially with $0.1 \mathrm{M}$ PBS and a fixative solution of precooled $4 \%$ paraformaldehyde, and brains were removed and immersion fixed in $4 \%$ paraformaldehyde. Frozen sections with a thickness of 5-10 $\mu \mathrm{m}$ were made by using a cryomicrotome (Thermo Fisher Scientific, Waltham, MA) for senescence-associated $\beta$-galactosidase (SA- $\beta$-gal) staining (Noren Hooten and Evans, 2017). After sectioning, tissues were covered with $500 \mu \mathrm{l}$ of precooled fixative solution for 15 minutes at room temperature. Then, the tissues were rinsed two times with PBS and incubated with $\beta$-galactosidase staining solution at $37^{\circ} \mathrm{C}$ overnight in a dry incubator (i.e., no $\mathrm{CO}_{2}$ ). The stained tissues were examined under a light microscope (model KS 300; Carl Zeiss Microscopy GmbH, Jena, Germany). For quantification, we used the ImageJ open source software to perform digital slide image counts.

Nissl Staining. Brains were fixed in $4 \%$ paraformaldehyde, embedded by paraffin, and cut into coronal $5-\mu \mathrm{m}$-thick sections for Nissl staining (Liu et al., 2015). In brief, the sections were deparaffinized in xylene and rehydrated using gradual alcohol, treated with Nissl staining solution (Solarbio; Beijing, People's Republic of China) for 5 minutes, and then mounted with neutral balsam. The hippocampal CA1 region was examined under a light microscope (Leica Microsystems Ltd., Wetzlar, Germany) by investigators who were blinded to the experimental groups. The numbers of Nissl bodies were captured in the three fields of the CA1 region of the hippocampus. For quantification, we used the ImageJ open source software to analyze.

Electron Microscopy. For the experiments with electron microscopy, animals randomly selected from each group were sacrificed and left hippocampal tissue samples were dissected, immediately put in $2.5 \%$ glutaraldehyde, and postfixed with $1 \% \mathrm{OsO}_{4}$. Tissue samples were dehydrated in a gradient series of diluted ethanol. After dehydration, the samples were infiltrated with a mixture of propylene oxide and epoxy resin (volume ratio, $1: 1$ ) at $70^{\circ} \mathrm{C}$ overnight. Ultra-thin sections (about $50 \mathrm{~mm}$ ) were cut, mounted on a copper grid, stained with uranyl acetate and lead citrate, and observed using a transmission electron microscope (model H-7650; Hitachi, Tokyo, Japan).

Western Blot. The hippocampus and cerebral cortex were sheared into small pieces, and the total protein was extracted 
using radioimmunoprecipitation assay lysis buffer. The supernatants were collected by centrifugation at $12,000 \mathrm{rpm}$ at $4^{\circ} \mathrm{C}$ for 20 minutes, and the protein concentration was measured using the BCA (bicinchoninic acid) Protein Assay Kit (Beyotime; Haimen, People's Republic of China). The equal amount of protein $(30 \mu \mathrm{g})$ was separated by $10 \%$ SDS-PAGE and transferred to $0.22 \mu \mathrm{m}$ polyvinylidene difluoride membranes (Millipore Shanghai Trading Company Ltd., Shanghai, People's Republic of China). The membranes were blocked in 5\% skim milk for 2 hours at room temperature. The membranes were then incubated at $4^{\circ} \mathrm{C}$ overnight with primary antibodies against p62 (1:1000 dilution; Abcam, Cambridge, UK), LC3-II (1:1000 dilution; Abcam), and $\beta$-actin (1:2000 dilution; Beyotime). After being washed, the polyvinylidene difluoride membranes were incubated with horseradish peroxidase-conjugated secondary antibodies for 2 hours at room temperature. The membrane-bound secondary antibody was detected using the ECL Select Kit (Beyotime) and was visualized using Gel Imaging (Bio-Rad, Hercules, CA).

Statistical Analysis. All results were analyzed by SPSS version 16.0 statistics software (IBM, Armonk, NY), and values were expressed as the mean \pm S.D. The escape latency in the MWM was analyzed with two-way analysis of variance and the Bonferroni test, and other results were analyzed by a one-way analysis of variance. $P<0.05$ was considered to be statistically significant.

\section{Results}

Effects of ICA on Learning and Memory Function of SAMP8 and SAMR1 Mice. A training trial lasted for 4 days, and the escape latency time was recorded. As shown in Fig. 1B, from day 2 to day 4, the mice in the SAMP8 group presented with a significantly prolonged escape latency compared with that of the SAMR1 group $(P<0.05)$, indicating the impairment of cognitive performance represented in mice in the SAMP8 group. However, the cognitive impairment of SAMP8 was improved after ICA administration, especially at the high dose of ICA $(80 \mathrm{mg} / \mathrm{kg})$ administered from day 3 to day $4(P<0.05)$. On the 5 th day, the time spent in the platform quadrant was recorded (Fig. 1C). Results showed that SAMP8 mice spent less time in the target quadrant than the SAMR1 mice $(P<0.05)$. The administration of ICA reduced the spatial memory impairment in a dose-dependent manner, particularly in the high-dose group $(P<0.05)$.

Effects of ICA on SA- $\beta$-Gal Activity in the Brains of SAMP8 and SAMR1 Mice. The SA- $\beta$-gal staining was used to evaluate the effects of ICA on the aging of brain tissue. The blue particles indicated SA- $\beta$-gal activity. As shown in Fig. $2 \mathrm{~A}$, the intensity of blue particles was increased in the brain region of SAMP8 mice, and mice in the SAMP8 group exhibited about 2-fold higher intensity of blue particles than SAMR1 mice $(P<0.05)$. However, a reduction in the positive expression of blue particles was observed in ICA treatment groups $(P<0.05)$, except for the mice receiving $20 \mathrm{mg} / \mathrm{kg}$ ICA (Fig. 2B). These results suggest that ICA is able to slow down cellular senescence in the brains of SAMP8 mice.

Effects of ICA on Hippocampal Neurons of SAMP8 and SAMR1 Mice. It has been documented that aging may cause neuronal atrophy or loss in the brain (Padurariu et al., 2012). To determine whether there is a loss of neuronal cell or structural alteration in the hippocampus of SAMP8 mice, Nissl staining was conducted to localize the cell body of neurons in the hippocampus. As shown in Fig. 3, the hippocampus CA1 region of mice in the SAMP8 group exhibited abnormal neurons and loss of Nissl bodies. The pyramidal layer of cells was significantly diminished with a marked reduction (79\%) in Nissl bodies in the hippocampus CA1 region in mice in the SAMP8 group compared with mice in the SAMR1 group. The treatment of ICA significantly inhibited neuronal loss and reversed neuronal structural changes in the hippocampi of SAMP8 mice $(P<0.05)$.

Effect of ICA on Autophagosome Formation in the Hippocampal Neurons of SAMP8 and SAMR1 Mice. Defects in autophagic activity are involved in a variety of neurodegenerative disorders (Son et al., 2012). Therefore, the

A
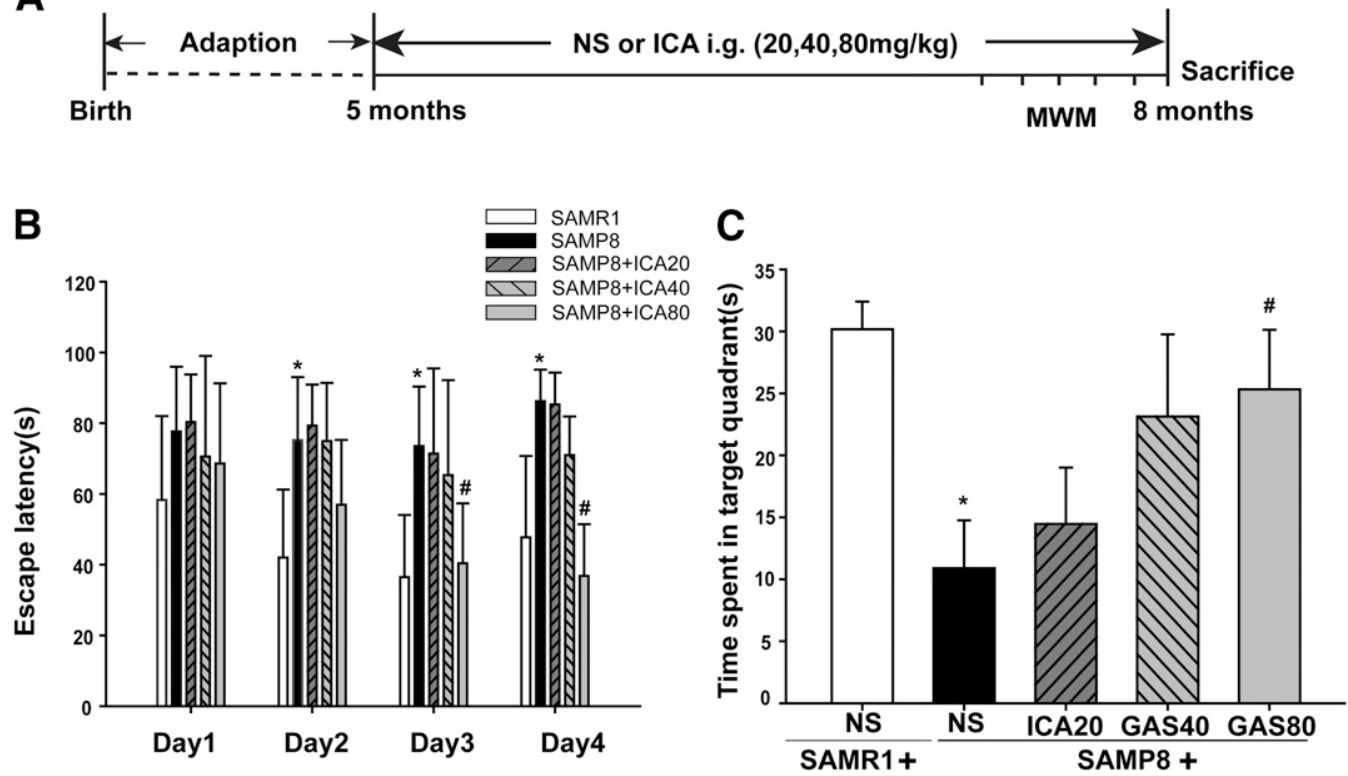

Fig. 1. Effects of ICA on MWM performance deficits in SAMP8 mice. (A) Schematic representation of the experimental design. (B) The latencies were measured to assess the mouse learning and memory ability in 4-day training trials. (C) The percentage of time spent in the target quadrant. Data are presented as the mean \pm S.D. $(n=8-12) . * P<0.05$ vs. SAMR1, $\# P<0.05$ vs. SAMP8. 

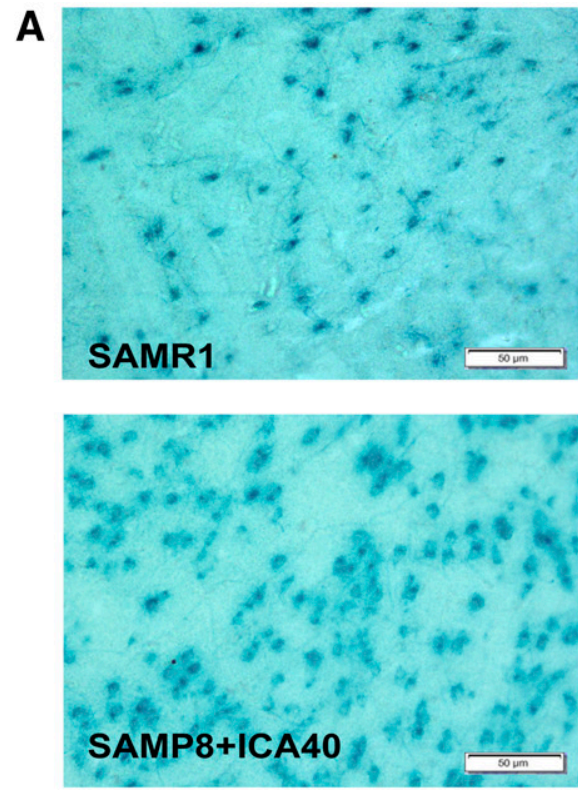
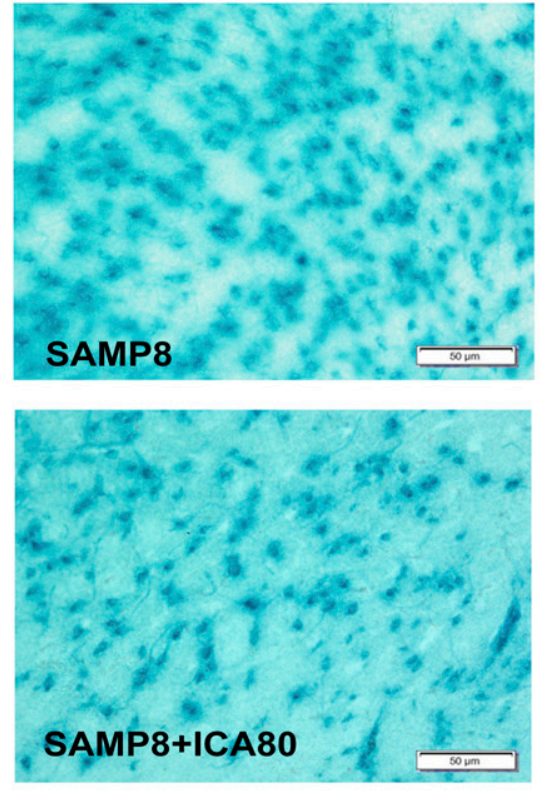
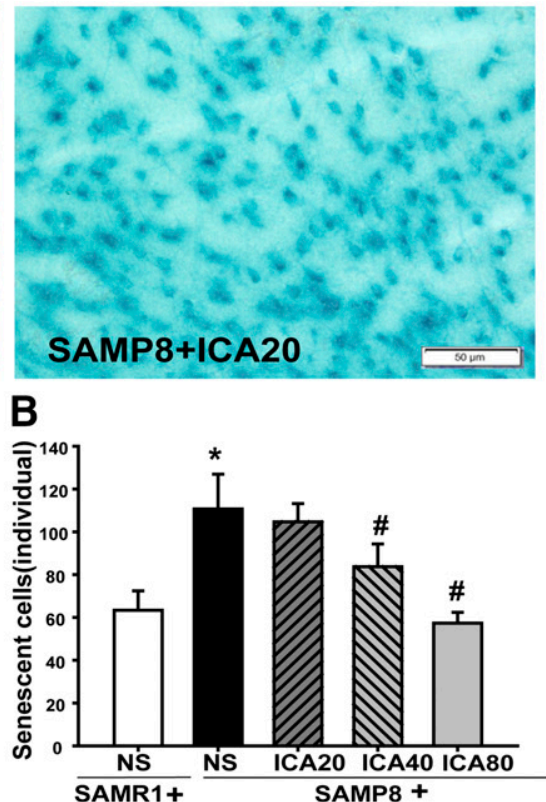

Fig. 2. Effects of ICA on SA- $\beta$-gal activity in brain tissue of SAMP8 and SAMR1 mice. (A) Representative images of SA- $\beta$-gal staining. Original magnification, $200 \times$. Scale bar, $50 \mu \mathrm{m}$. (B) Quantitation of senescent-positive cells. Data were expressed as the mean \pm S.D. $(n=4)$. $* P<0.05$ vs. SAMR1, $\# P<0.05$ vs. SAMP8.

hippocampal neuronal ultrastructure was examined by transmission electron microscopy. The observation revealed that, compared with SAMR1 mice, the hippocampal neurons in the brains of SAMP8 mice harbored disorganized and swollen endoplasmic reticulum. In addition, more autophagosomes were observed in hippocampal neurons of SAMP8 mice (Fig. 4, $\mathrm{Aa}$ and $\mathrm{Bb}$ ). After the administration of ICA, the number of autophagosomes was significantly decreased, accompanied by organized endoplasmic reticulum present in hippocampal neurons (Fig. 4, Cc, Dd, and Ee).
The Expression of LC3-II and p62 in the Hippocampus and Cortex of SAMP8 and SAMR1 Mice. To define a possible role of autophogosome formation in the aging of SAMP8 mice, the protein expressions of autophagic markers LC3-II and p62 in the hippocampus and cortex were examined by Western blot. As shown in Fig. 5, there was a significant increase in the expression levels of LC3-II and p62 in the hippocampus and cortex of mice in the SAMP8 group compared with those in the SAMR1 group $(P<0.05)$. After receiving 3 months of ICA treatment, the expression of LC3-II and p62
A
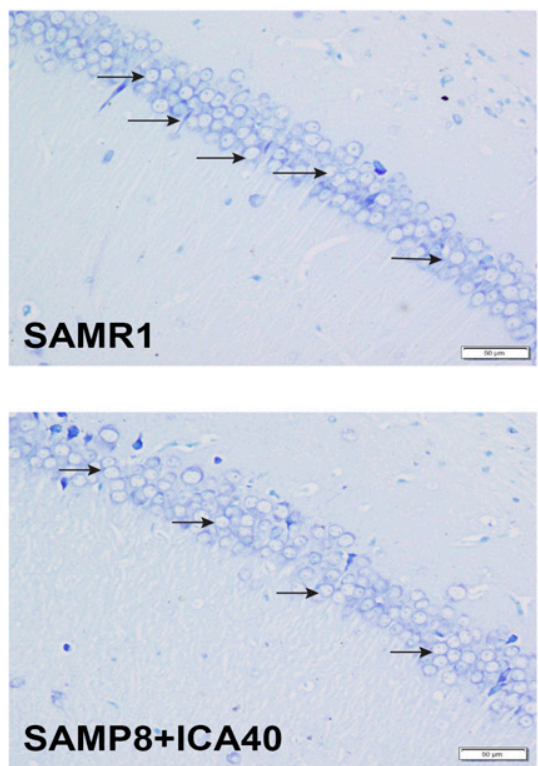
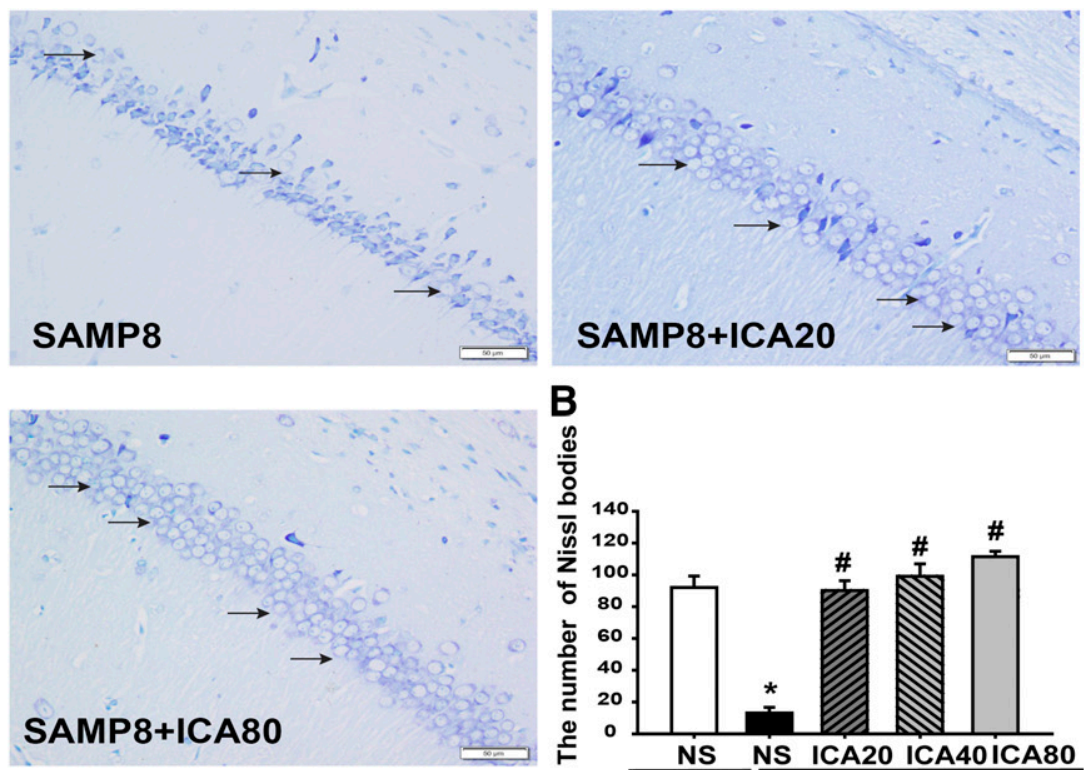

Fig. 3. Effect of ICA on hippocampal neurons of SAMP8 mice. (A) Representative images showing Nissl bodies in the hippocampal CA1. Original magnification, $200 \times$. Scale bar, $50 \mu \mathrm{m}$. (B) Quantitation of pyramidal cells in the CA1 hippocampal region. The numbers of Nissl bodies were captured in the three fields of the CA1 region of the hippocampus. The data represent the mean \pm S.D. $(n=4) . * P<0.01$ vs. SAMR1; \#P<0.01 vs. SAMP8. 


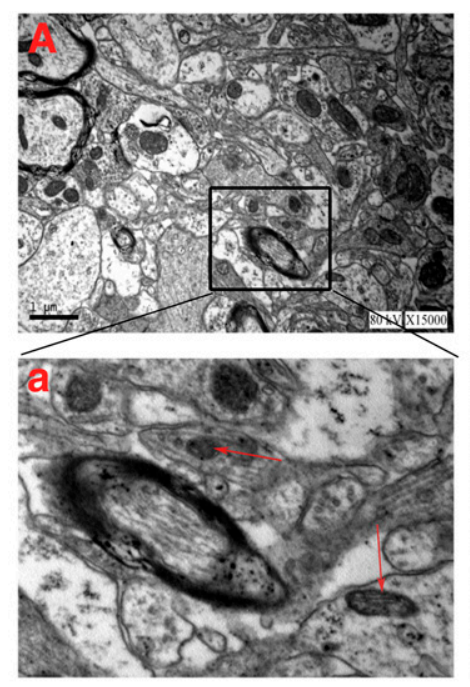

SAMR1

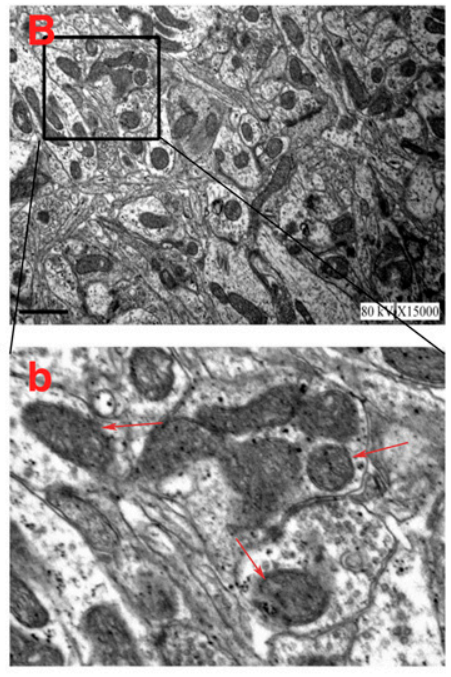

SAMP8

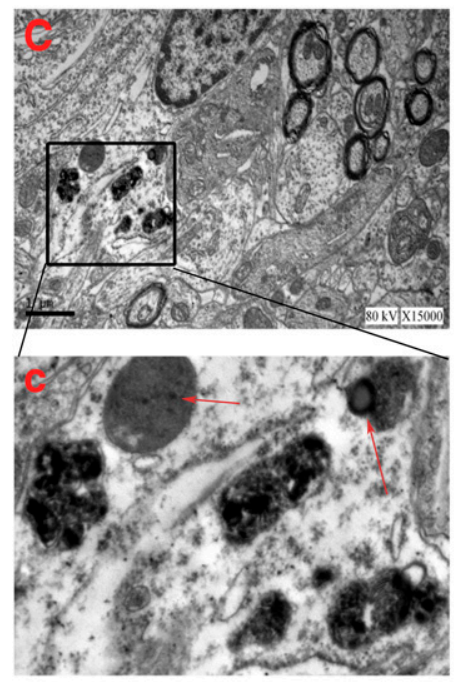

SAMP8+ICA20

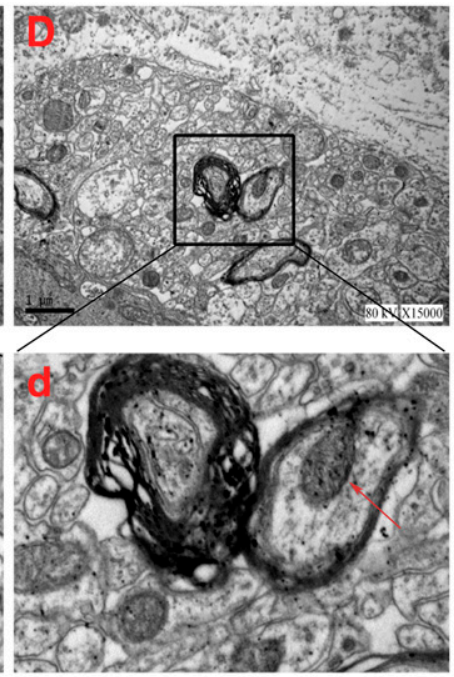

SAMP8+ICA40

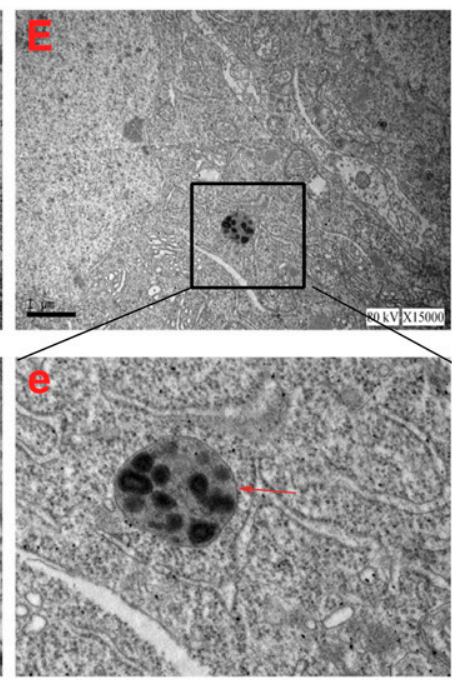

SAMP8+ICA80

Fig. 4. Effect of ICA on autophagosome formation in hippocampal neurons. A-E. Representative ultrastructure images of each group (magnification, $15,000 \times$; scale bar, $1 \mu \mathrm{m}$ ); a-e. Representative ultrastructure images of each group represent (magnification, $30,000 \times$; scale bar, $2 \mu \mathrm{m}$ ). The red arrows indicate autophagosomes $(n=4)$.

was reduced $(P<0.05)$, indicating the inhibitory effect of ICA on autophagosome formations in SAMP8 mice.

\section{Discussion}

The present study revealed that ICA treatment effectively attenuated cognitive deficits, reduced the number of senescence cells, inhibited neuronal loss, and improved neuronal structural changes in the brains of SAMP8 mice. Simultaneously, ICA treatment also decreased the formation of autophagosomes in the hippocampus of SAMP8 mice, and reduced the expression of autophagy-related proteins LC3-II and p62. This study is among the first to demonstrate ICA regulation of autophagy in SAMP8 mice.

SAMP8 mice are an excellent brain-aging model of learning and memory defects (Morley et al., 2012; Akiguchi et al., 2017). Synaptic loss and cognitive deficits were increased significantly in 8-month-old SAMP8 mice (Chen et al., 2014b). Consistent with these reports, our present study showed that learning and memory were significantly impaired in 8-monthold SAMP8 mice compared with the control strain SARM1 mice. The cognitive impairments of SAMP8 mice were significantly improved after the administration of ICA for 3 months, especially at the dose of $80 \mathrm{mg} / \mathrm{kg}$, in agreement with the literature (He et al., 2010). Additionally, SAMP8 mice showed increased anxiety-like behavior compared with SAMR1 mice (Meeker et al., 2013), and ICA has been shown to have antianxiety effects (Li et al., 2014; Xiao et al., 2016), which could also contribute to cognition improvement.

Cellular senescence plays an important role in brain aging. An increased number of senescent cells was observed in the lateral subventricular, hippocampus and cortical regions of aging mice (Geng et al., 2010; Carnero, 2013; Shimabukuro et al., 2016). SA- $\beta$-gal is a hydrolase enzyme to catalyze the hydrolysis of $\beta$-galactosidase into monosaccharides only in senescent cells, and is regarded as a biomarker of cellular senescence (Itahana et al., 2007). The SA- $\beta$-gal staining assay has been widely used as biologic indicators for aging cells, and 
A

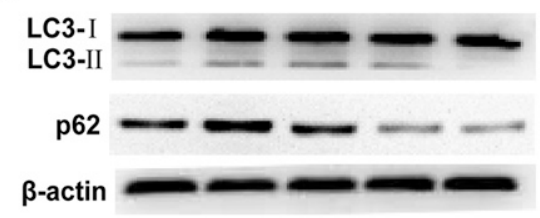

C

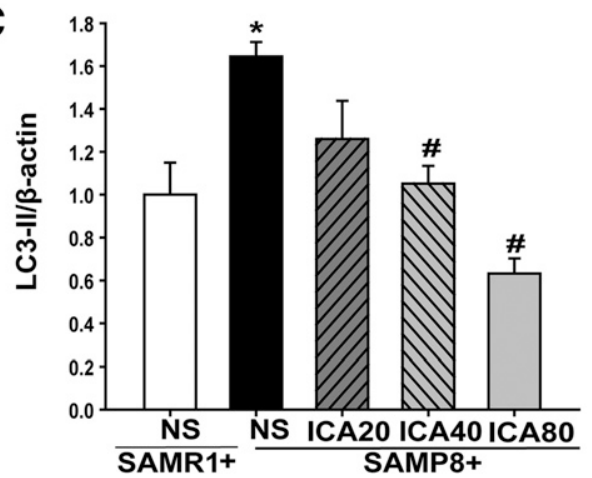

E

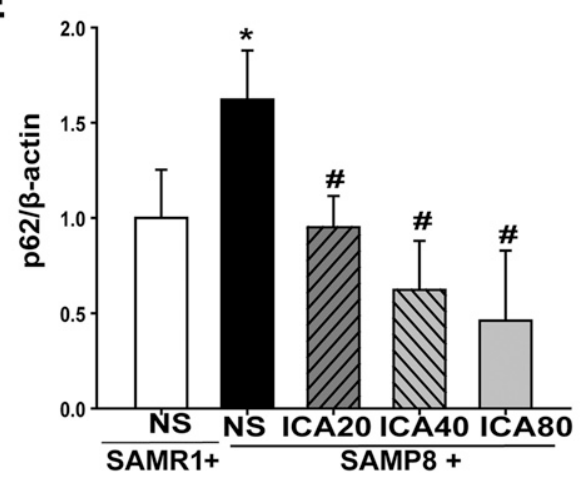

B

LC3-I LC3-II

p62

$\beta$-actin

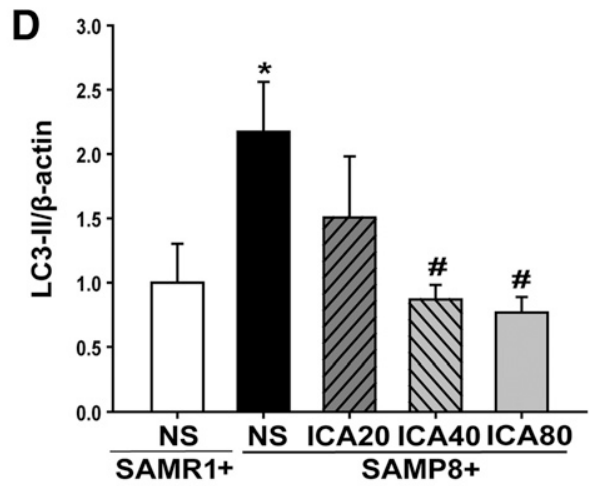

$\mathbf{F}$

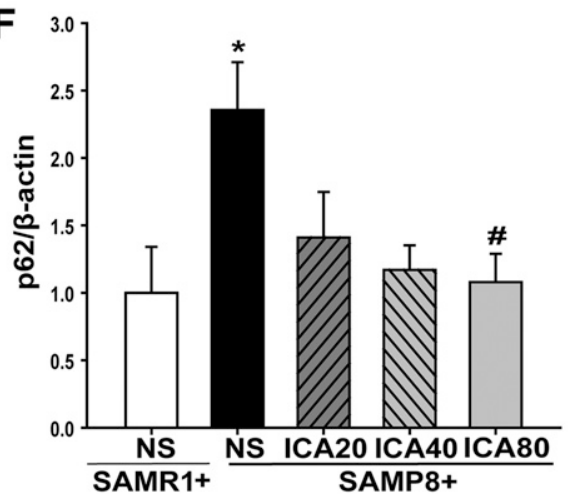

Fig. 5. Effect of ICA on the expression of LC3-II and p62 in hippocampus and cortex. (A) Representative bands of protein expression in hippocampus. (B) Representative bands of protein expression in cortex. (C) Quantitation of LC3-II protein level in hippocampus. (D) Quantitation of LC3-II protein level in cortex. (E) Quantitation of p62 protein level in hippocampus. (F) Quantitation of $\mathrm{p} 62$ protein level in cortex. The relative optical density was normalized to $\beta$-actin. The data were expressed as the mean \pm S.D. $(n=4) . * P<0.05$ vs. SAMR1; $\# P<0.05$ vs. SAMP8. senescent cells are stained with blue precipitate in the cytoplasm (Zhu et al., 2014). Therefore, we further explored the effect of ICA on aging using SA- $\beta$-gal assay to stain senescent cells in the cortex. Our results clearly demonstrated that 8-month-old SAMP8 mice receiving ICA treatment exhibited significantly decreased SA- $\beta$-gal stain-positive aging cells in the cortex compared with untreated SAMP8 mice. These findings indicated that the attenuation of senescent cells may contribute to the protective ability of ICA against brain aging.

The hippocampus is an important part of the limbic system in the brain; to be more precise, emotion and cognition, as well as aging, are all closely related to hippocampal functions (Simic et al., 1997). Particularly, the hippocampus is vital for spatial learning and memory, and age-related progressive neuronal damage in the hippocampus can lead to cognitive impairment (Chen et al., 1998; Borgesius et al., 2011; Thongasa et al., 2013). In addition, most studies have demonstrated that the hippocampus is very sensitive to aging and is the first affected organ in terms of morphology and physiology in the aging process (West et al., 1994; Bhatnagar et al., 1997; Onozuka et al., 2002; Watanabe et al., 2002). To obtain more insight into the mechanism of how ICA improves cognitive function and reverses the aging process in SAMP8 mice, we further examined the structure of neuronal cells in the hippocampal CA1 region. As shown in Fig. 3B, the hippocampus CA1 region of SAMP8 mice exhibited abnormal neurons and loss of Nissl bodies. Electron microscopy revealed disorganized endoplasmic reticuli in the hippocampi of 8-month-old SAMP8 mice (Fig. 4Bb). All of these results further confirmed the damage and loss of neurons in the brains of aging SAMP8 mice because neuronal loss in the hippocampal CA1 subregion has been identified as a memory-associated neuropathological marker in both humans and animals, and the loss of neurons in the CA1 area occurs as age advances (Markham et al., 2005; Banji et al., 2013; Hosseini-sharifabad and Esfandiari, 2015). Strikingly, all of these lesions and the loss of neurons were significantly reduced in SAMP8 mice receiving ICA treatment for 3 months.

Importantly, we demonstrated that increased autophagosome formation in hippocampal neurons occurred in the 8-month-old SAMP8 mice, compared with age-matched SAMR1 mice, supporting the notion that alterations in autophagy occur in the aging process of SAMP8 mice (Ma et al., 2011; Chen et al., 2014b). Autophagy is a self-degradation pathway to eliminate damaged organelles (Mathiassen et al., 2017). However, autophagy is a double-edged sword and overinduction of autophagy may cause neuronal cell death. Aging is characterized by abnormal aggregation of proteins in brain neurons, triggering the induction of autophagy (Tan et al., 2014). Previous studies also reported that autophagic 
vacuoles were observed in the hippocampi of patients with Alzheimer's disease (Nixon et al., 2005). In the present study, electron microscopy revealed an aging-dependent increase in autophagosome formation in the hippocampi of SMAP8 mice, which was inhibited by ICA. Furthermore, we also examined LC3 and p62 changes in the hippocampus and cortex, and found that the LC3-II expression showed an increase in hippocampi and cortices of 8-month-old SAMP8 mice, which is consistent with previous findings (Ma et al., 2011; Chen et al., 2014b). After the administration of ICA, the expression of LC3-II decreased. Besides, the protein expression of p62, a marker of autophagy, was increased in SAMP8 mice, and diminished after the administration of ICA. These results suggest that ICA could increase autophagosome-lysosome fusion, thus reducing the autophagy flux, in agreement with the antiautophagy effects of ICA in the literature (Algandaby et al., 2017; Li et al., 2017).

In conclusion, ICA has beneficial effects on improving learning and memory impairment, reducing the number of senescence cells, inhibiting neuronal loss, and reversing neuronal structural changes in SAMP8 mice through a mechanism that may be related to the regulation of autophagy.

\section{Authorship Contributions}

Participated in research design: $\mathrm{Wu}, \mathrm{Xu}$, and Shi.

Conducted experiments: Chen, $\mathrm{Wu}$, and $\mathrm{Xu}$.

Performed data analysis: Chen, B. Liu, Wu, and Shi.

Wrote or contributed to the writing of the manuscript: Chen, B. Liu, J. Liu, Zhou, and Shi.

\section{References}

Akiguchi I, Pallàs M, Budka H, Akiyama H, Ueno M, Han J, Yagi H, Nishikawa T, Chiba Y, Sugiyama H, et al. (2017) SAMP8 mice as a neuropathological model of accelerated brain aging and dementia: Toshio Takeda's legacy and future directions. Neuropathology 37:293-305.

Algandaby MM, Breikaa RM, Eid BG, Neamatallah TA, Abdel-Naim AB, and Ashour OM (2017) Icariin protects against thioacetamide-induced liver fibrosis in rats: implication of anti-angiogenic and anti-autophagic properties. Pharmacol Rep 69: 616-624.

Banji D, Banji OJ, Dasaroju S, and Annamalai AR (2013) Piperine and curcumin exhibit synergism in attenuating D-galactose induced senescence in rats. Eur $J$ Pharmacol 703:91-99.

Barzilai N, Cuervo AM, and Austad S (2018) Aging as a biological target for prevention and therapy. JAMA 320:1321-1322.

Bhatnagar M, Cintra A, Chadi G, Lindberg J, Oitzl M, De Kloet ER, Möller A, Agnat LF, and Fuxe K (1997) Neurochemical changes in the hippocampus of the brown Norway rat during aging. Neurobiol Aging 18:319-327.

Borgesius NZ, de Waard MC, van der Pluijm I, Omrani A, Zondag GC, van der Horst GT, Melton DW, Hoeijmakers JH, Jaarsma D, and Elgersma Y (2011) Accelerated age-related cognitive decline and neurodegeneration, caused by deficient DNA repair. J Neurosci 31:12543-12553.

Carnero A (2013) Markers of cellular senescence. Methods Mol Biol 965:63-81.

Chen M, Hao J, Yang Q, and Li G (2014a) Effects of icariin on reproductive functions in male rats. Molecules 19:9502-9514.

Chen Y, Wei G, Nie H, Lin Y, Tian H, Liu Y, Yu X, Cheng S, Yan R, Wang Q, et al. (2014b) $\beta$-Asarone prevents autophagy and synaptic loss by reducing ROCK expression in asenescence-accelerated prone 8 mice. Brain Res 1552:41-54.

Chen YC, Lei JL, Chen QS, and Wang SL (1998) Effect of physical training on the age-related changes of acetylcholinesterase-positive fibers in the hippocampal formation and parietal cortex in the C57BL/6J mouse. Mech Ageing Dev 102:81-93.

Cuesta S, Kireev R, García C, Rancan L, Vara E, and Tresguerres JA (2013) Melatonin can improve insulin resistance and aging-induced pancreas alterations in senescence-accelerated prone male mice (SAMP8). Age (Dordr) 35:659-671.

Deak F, Freeman WM, Ungvari Z, Csiszar A, and Sonntag WE (2016) Recent developments in understanding brain aging: implications for Alzheimer's disease and vascular cognitive impairment. J Gerontol A Biol Sci Med Sci 71:13-20.

De Rechter S, Decuypere JP, Ivanova E, van den Heuvel LP, De Smedt H, Levtchenko E, and Mekahli D (2016) Autophagy in renal diseases. Pediatr Nephrol 31 737-752.

Fang EF, Scheibye-Knudsen M, Jahn HJ, Li J, Ling L, Guo H, Zhu X, Preedy V, Lu H, Bohr VA, et al. (2015) A research agenda for aging in China in the 21st century. Ageing Res Rev 24 (Pt B):197-205.

Farr SA, Niehoff ML, Ceddia MA, Herrlinger KA, Lewis BJ, Feng S, Welleford A, Butterfield DA, and Morley JE (2016) Effect of botanical extracts containing carnosic acid or rosmarinic acid on learning and memory in SAMP8 mice. Physiol Behav 165 $328-338$.
Farr SA, Price TO, Banks WA, Ercal N, and Morley JE (2012) Effect of alpha-lipoic acid on memory, oxidation, and lifespan in SAMP8 mice. J Alzheimers Dis 32 : $447-455$

García-Matas S, Paul RK, Molina-Martínez P, Palacios H, Gutierrez VM, Corpas R, Pallas M, Cristòfol R, de Cabo R, and Sanfeliu C (2015) In vitro caloric restriction induces protective genes and functional rejuvenation in senescent SAMP8 astrocytes. Aging Cell 14:334-344.

Geng YQ, Guan JT, Xu XH, and Fu YC (2010) Senescence-associated betagalactosidase activity expression in aging hippocampal neurons. Biochem Biophys Res Commun 396:866-869.

Guo J, Li F, Wu Q, Gong Q, Lu Y, and Shi J (2010) Protective effects of icariin on brain dysfunction induced by lipopolysaccharide in rats. Phytomedicine 17: $950-955$.

He XL, Zhou WQ, Bi MG, and Du GH (2010) Neuroprotective effects of icariin on memory impairment and neurochemical deficits in senescence-accelerated mouse prone 8 (SAMP8) mice. Brain Res 1334:73-83.

Hosseini-sharifabad M and Esfandiari E (2015) Effect of Boswellia serrata gum resin on the morphology of hippocampal CA1 pyramidal cells in aged rat. Anat Sci Int 90 $47-53$

Itahana K, Campisi J, and Dimri GP (2007) Methods to detect biomarkers of cellular senescence: the senescence-associated beta-galactosidase assay. Methods Mol Biol 371:21-31.

Jin F, Gong QH, Xu YS, Wang LN, Jin H, Li F, Li LS, Ma YM, and Shi JS (2014) Icariin, a phosphodiesterase-5 inhibitor, improves learning and memory in APP/ PS1 transgenic mice by stimulation of NO/cGMP signalling. Int $J$ Neuropsychopharmacol 17:871-881.

Kang C and Elledge SJ (2016) How autophagy both activates and inhibits cellular senescence. Autophagy 12:898-899.

Li B, Duan X, Xu C, Wu J, Liu B, Du Y, Luo Q, Jin H, Gong W, and Dong J (2014) Icariin attenuates glucocorticoid insensitivity mediated by repeated psychosocial stress on an ovalbumin-induced murine model of asthma. Int Immunopharmacol 19:381-390.

Li C, Li Q, Mei Q, and Lu T (2015) Pharmacological effects and pharmacokinetic properties of icariin, the major bioactive component in Herba Epimedii. Life Sci 126:57-68.

Li D, Ke Y, Zhan R, Liu C, Zhao M, Zeng A, Shi X, Ji L, Cheng S, Pan B, et al. (2018) Trimethylamine-N-oxide promotes brain aging and cognitive impairment in mice. Aging Cell 17:e12768.

Li F, Dong HX, Gong QH, Wu Q, Jin F, and Shi JS (2015) Icariin decreases both APP and $\mathrm{A} \beta$ levels and increases neurogenesis in the brain of Tg2576 mice. Neuroscience 304:29-35.

Li H, Yuan Y, Zhang Y, Zhang X, Gao L, and Xu R (2017) Icariin inhibits AMPKdependent autophagy and adipogenesis in adipocytes in vitro and in a model of Graves' Orbitopathy in vivo. Front Physiol 8:45.

Liu B, Gao JM, Li F, Gong QH, and Shi JS (2018) Gastrodin attenuates bilateral common carotid artery occlusion-induced cognitive deficits via regulating $A \beta$ related proteins and reducing autophagy and apoptosis in rats. Front Pharmacol 9:405.

Liu H, Deng Y, Gao J, Liu Y, Li W, Shi J, and Gong Q (2015) Sodium hydrosulfide attenuates beta-amyloid-induced cognitive deficits and neuroinflammation via modulation of MAPK/NF-кB pathway in rats. Curr Alzheimer Res 12:673-683.

López-Otín C, Blasco MA, Partridge L, Serrano M, and Kroemer G (2013) The hallmarks of aging. Cell 153:1194-1217.

Ma Q, Qiang J, Gu P, Wang Y, Geng Y, and Wang M (2011) Age-related autophagy alterations in the brain of senescence accelerated mouse prone 8 (SAMP8) mice. Exp Gerontol 46:533-541.

Manich G, Mercader C, del Valle J, Duran-Vilaregut J, Camins A, Pallàs M, Vilaplana J, and Pelegrí C (2011) Characterization of amyloid- $\beta$ granules in the hippocampus of SAMP8 mice. $J$ Alzheimers Dis 25:535-546.

Markham JA, McKian KP, Stroup TS, and Juraska JM (2005) Sexually dimorphic aging of dendritic morphology in CA1 of hippocampus. Hippocampus 15:97-103.

Mathiassen SG, De Zio D, and Cecconi F (2017) Autophagy and the cell cycle: a complex landscape. Front Oncol 7:51.

Mecocci P, Boccardi V, Cecchetti R, Bastiani P, Scamosci M, Ruggiero C, and Baron $\mathrm{M}$ (2018) A long journey into aging, brain aging, and Alzheimer's disease following the oxidative stress tracks. J Alzheimers Dis 62:1319-1335.

Meeker HC, Chadman KK, Heaney AT, and Carp RI (2013) Assessment of social interaction and anxiety-like behavior in senescence-accelerated-prone and -resistant mice. Physiol Behav 118:97-102.

Mo ZT, Li WN, Zhai YR, and Gong QH (2016) Icariin attenuates OGD/R-Induced autophagy via Bcl-2-dependent cross talk between apoptosis and autophagy in PC12 cells. Evid Based Complement Alternat Med 2016:4343084.

Morley JE, Farr SA, Kumar VB, and Armbrecht HJ (2012) The SAMP8 mouse: a model to develop therapeutic interventions for Alzheimer's disease. Curr Pharm Des 18:1123-1130.

Nixon RA, Wegiel J, Kumar A, Yu WH, Peterhoff C, Cataldo A, and Cuervo AM (2005) Extensive involvement of autophagy in Alzheimer disease: an immunoelectron microscopy study. J Neuropathol Exp Neurol 64:113-122.

Noren Hooten N and Evans MK (2017) Techniques to induce and quantify cellular senescence. J Vis Exp (123):e55533.

Onozuka M, Watanabe K, Fujita M, Tonosaki K, and Saito S (2002) Evidence for involvement of glucocorticoid response in the hippocampal changes in aged molarless SAMP8 mice. Behav Brain Res 131:125-129.

Padurariu M, Ciobica A, Mavroudis I, Fotiou D, and Baloyannis S (2012) Hippocampal neuronal loss in the CA1 and CA3 areas of Alzheimer's disease patients. Psychiatr Danub 24:152-158.

Serrano-Pozo A, Frosch MP, Masliah E, and Hyman BT (2011) Neuropathological alterations in Alzheimer disease. Cold Spring Harb Perspect Med 1:a006189. Shimabukuro MK, Langhi LG, Cordeiro I, Brito JM, Batista CM, Mattson MP, and Mello Coelho Vd (2016) Lipid-laden cells differentially distributed in the aging 
brain are functionally active and correspond to distinct phenotypes. Sci Rep 6: 23795.

Simić G, Kostović I, Winblad B, and Bogdanović N (1997) Volume and number of neurons of the human hippocampal formation in normal aging and Alzheimer's disease. J Comp Neurol 379:482-494.

Simonsen A, Cumming RC, Brech A, Isakson P, Schubert DR, and Finley KD (2008) Promoting basal levels of autophagy in the nervous system enhances longevity and oxidant resistance in adult Drosophila. Autophagy 4:176-184.

Son JH, Shim JH, Kim KH, Ha JY, and Han JY (2012) Neuronal autophagy and neurodegenerative diseases. Exp Mol Med 44:89-98.

Tan CC, Yu JT, Tan MS, Jiang T, Zhu XC, and Tan L (2014) Autophagy in aging and neurodegenerative diseases: implications for pathogenesis and therapy. Neurobiol Aging 35:941-957.

Thong-asa K, Chompoopong S, Tantisira MH, and Tilokskulchai K (2013) Reversible short-term and delayed long-term cognitive impairment induced by chronic mild cerebral hypoperfusion in rats. $J$ Neural Transm (Vienna) 120:1225-1235.

Tsuduki T, Honma T, Nakagawa K, Ikeda I, and Miyazawa T (2011) Long-term intake of fish oil increases oxidative stress and decreases lifespan in senescenceaccelerated mice. Nutrition 27:334-337.

Wang GQ, Li DD, Huang C, Lu DS, Zhang C, Zhou SY, Liu J, and Zhang F (2018) Icariin reduces dopaminergic neuronal loss and microglia-mediated inflammation in vivo and in vitro. Front Mol Neurosci 10:441.
Wang Y, Ma Q, Ma X, Zhang Z, Liu N, and Wang M (2017) Role of mammalian target of rapamycin signaling in autophagy and the neurodegenerative process using a senescence accelerated mouse-prone 8 model. Exp Ther Med 14:1051-1057.

Watanabe K, Ozono S, Nishiyama K, Saito S, Tonosaki K, Fujita M, and Onozuka M (2002) The molarless condition in aged SAMP8 mice attenuates hippocampal Fos induction linked to water maze performance. Behav Brain Res 128:19-25.

West MJ, Coleman PD, Flood DG, and Troncoso JC (1994) Differences in the pattern of hippocampal neuronal loss in normal ageing and Alzheimer's disease. Lancet 344:769-772.

Xiao H, Wignall N, and Brown ES (2016) An open-label pilot study of icariin for co-morbid bipolar and alcohol use disorder. Am J Drug Alcohol Abuse 42:162-167. Zhu J, Mu X, Zeng J, Xu C, Liu J, Zhang M, Li C, Chen J, Li T, and Wang Y (2014) Ginsenoside Rg1 prevents cognitive impairment and hippocampus senescence in a rat model of D-galactose-induced aging. PLoS One 9:e101291.

Address correspondence to: Dr. Jing-Shan Shi, Key Laboratory of Basic Pharmacology of Ministry of Education and Joint International Research Laboratory of Ethnomedicine of Ministry of Education, Zunyi Medical University, Zunyi 563000, People's Republic of China. E-mail: shijingshan2018@ 163.com 